\title{
Germanica
}

\section{Made in Germany. Notes sur l'histoire de la bande dessinée en Allemagne}

Made in Germany. Notizen zur Geschichte des Comics in Deutschland.

Made in Germany. The evolution of comics in Germany.

\section{Andreas C. Knigge}

Traducteur : Annick Carlier

\section{OpenEdition}

\section{Journals}

Édition électronique

URL : http://journals.openedition.org/germanica/1082

DOI : 10.4000/germanica. 1082

ISSN : 2107-0784

Éditeur

Université de Lille

Édition imprimée

Date de publication : 31 décembre 2010

Pagination : 11-24

ISBN : 9782913857261

ISSN : 0984-2632

\section{Référence électronique}

Andreas C. Knigge, "Made in Germany. Notes sur l'histoire de la bande dessinée en Allemagne »,

Germanica [En ligne], 47 | 2010, mis en ligne le 01 décembre 2012, consulté le 06 octobre 2020. URL

http://journals.openedition.org/germanica/1082 ; DOI : https://doi.org/10.4000/germanica.1082

Ce document a été généré automatiquement le 6 octobre 2020.

(C) Tous droits réservés 


\title{
Made in Germany. Notes sur l'histoire de la bande dessinée en Allemagne
}

\author{
Made in Germany. Notizen zur Geschichte des Comics in Deutschland. \\ Made in Germany. The evolution of comics in Germany.
}

\author{
Andreas C. Knigge
}

Traduction : Annick Carlier

1

1 L'Allemagne ne fait pas partie de ces pays qui, en Europe même, comme la France, la Belgique, l'Espagne, l'Italie, sans compter la Grande-Bretagne, peuvent se réclamer d'une longue tradition de la bande dessinée. Abstraction faite de travaux isolés de quelques rares artistes, la BD ne commença à se développer qu'après la Seconde Guerre mondiale et avec la division de l'Allemagne. Le genre évolua d'ailleurs de façon très différente à l'Ouest et à l'Est. Tandis qu'en Allemagne de l'Ouest des traductions provenant essentiellement de l'espace franco-belge et des USA dominèrent très vite le marché, la RDA, s'inscrivant dans la perspective d'une éducation socialiste, prit le parti de produire elle-même sa propre littérature de bande dessinée. Le magazine Mosaik qui tirait chaque mois à quasiment un million d'exemplaires et qui continue de paraître, devint vite en Allemagne de l'Est l'organe central d'une culture par le dessin à destination de la jeunesse ; avec Micky Maus elle est aujourd'hui la revue de BD la plus ancienne dans l'Allemagne unifiée. Ce n'est qu'après l'unification allemande en 1989 qu'apparut une nouvelle génération de dessinateurs allemands qui sont l'objet d'un intérêt grandissant hors des frontières et qui sont publiés en traduction à l'étranger.

2 Cependant, pour faire l'historique de la bande dessinée allemande, il faut commencer par évoquer les débuts de ce genre. Les Américains ont de fait pour référence Yellow Kid de Richard F. Outcault, les Français, les nouvelles illustrées de Rodolphe Töpffer, et les 
Anglais, Ally Sloper; s'il faut situer les débuts de la BD allemande en tant que médium narratif graphique, on pense naturellement au virtuose de l'image grâce à qui l'histoire illustrée classique, qui apparut partout en Europe au milieu du XIX ${ }^{\mathrm{e}}$ siècle, connut son apogée : Wilhelm Busch (1832-1908) peut être effectivement considéré comme l'ancêtre de la bande dessinée ; mais tout dépend de ce que l'on entend par bande dessinée. Sur le procédé de la narration sous forme de succession d'images, le Suisse Töpffer le devançait largement. Et c'est Outcault qui, le premier, - le 25 octobre 1895 dans le supplément du dimanche du New York Journal - associa à une succession d'images des bulles, qui jusqu'à ce jour n'étaient utilisées que dans les caricatures et non dans des histoires illustrées.

\section{2}

3 Il semble cependant que ce soient surtout des éléments techniques d'impression qui aient empêché Busch de faire le pas pour passer de l'histoire illustrée à la bande dessinée moderne. Dans la collection du musée Wilhelm Busch à Hanovre se trouve un feuillet d'un grand format $(49 \times 22 \mathrm{~cm})$ ayant pour titre Der böse Hundsfänger und das arme Hündlein, qui doit dater d'entre 1864 et 1866 et sur lequel on peut apercevoir l'esquisse d'une histoire illustrée que Busch n'a jamais achevée sous cette forme (il en a simplement utilisé des motifs à l'automne 1866 pour ses Münchener Bilderbögen 431 et 432). Rétrospectivement, ce feuillet (une pièce unique dans ses œuvres posthumes), est tout à fait extraordinaire pour la façon dont Busch l'a agencé pour y insérer son histoire. Contrairement à la pratique en usage à cette époque, les formats des images y sont déterminés par l'espace que sous-tend l'action; ainsi l'ensemble des douze images sont de largeur différente et viennent s'agencer sur une planche au format plus long que large en trois bandes superposées, les textes en prose se situant en dessous du dessin. Ce qui est particulièrement frappant est le caractère dense, à la manière d'un film presque, de la succession de chacune des scènes illustrées qui ne sont pas séparées entre elles par de grands espaces vides comme le voudrait l'esprit de l'époque, mais au contraire par de minces lignes entourant chacune des illustrations. On trouve entre deux images, pour les démarquer, un mince filet blanc.

4 Ainsi Busch a-t-il préfiguré un concept graphique qui devait devenir, trente ans plus tard seulement, une bande dessinée dans les suppléments du dimanche de journaux américains. L'éditeur de Busch, Kaspar Braun, ne précisa pas à ce dernier la forme qu'il donnerait à ses histoires pour les publier : ainsi dans la revue satirique Fliegende Blätter, les pages de texte étant organisées en deux colonnes, les images furent-elles reproduites l'une en dessous de l'autre tandis que dans le Münchener Bilderbogen, elles se retrouvèrent disposées horizontalement. Elles pouvaient aussi être publiéessous forme de livre. Toutes les réflexions de Busch sur une composition d'ensemble de ses pages furent ainsi réduites à néant; il transmit à Braun ses dessins sous une forme décousue et sur des feuilles numérotées et s'en remit à son éditeur pour la composition finale.

5 Le histoires illustrées de Busch parurent également assez vite, et encore de son vivant, dans de nombreuses traductions. Elles parurent même en hébreu, en russe et en japonais. Lui même appelait son art " écrire en images », considérant " d'abord l'image, ensuite le texte », les deux devant « se fondre intimement l'un dans l'autre ». D'un trait maîtrisé, il sut donner vie à des caractères singuliers et développa un don étonnant à la fois comme dessinateur et comme auteur de textes ciselés. L'ironie cachée que 
véhiculent ses vers n'apparaît qu'avec le contraste généré par les dessins correspondants, l'humour véritable de Busch opérant avant tout dans ce jeu d'étroite correspondance entre l'image et le texte, une association qui est totalement nouvelle chez lui. De surcroit, en appliquant le "principe de la jubilation maligne", un motif constant que l'on retrouve dans ses histoires illustrées, il a considérablement influencé thématiquement les premières BD de presse qui apparurent au tournant du siècle dans les quotidiens new-yorkais.

6 Selon une anecdote légendaire, William R. Hearst, en réaction au succès du Yellow Kid de son concurrent Joseph Pulitzer, et après avoir introduit de nouveaux comic-strips dans les suppléments du dimanche de ses quotidiens, fit appel au dessinateur Rudolph Dirks, originaire d'Allemagne du Nord, pour produire something like Max and Moritz. C'est pour obéir à cette injonction que Dirks commença en 1897 ses Katzenjammer Kids, la plus ancienne bande dessinée de l'histoire du genre à paraître encore aujourd'hui. Dirks avait voulu au début se démarquer de Busch en introduisant un troisième larron, mais, par la suite, sur le conseil de Hearst, le frère surnuméraire avait disparu pour ne plus jamais apparaître. Le fait que les Katzenjammer Kids furent publiés immanquablement sous le titre Max und Moritz, dans le Morgen Journal de Hearst, une édition en langue allemande de son New York Journal (les Allemands constituaient à l'époque, après les Irlandais, la plus importante communauté d'immigrés, atteste de l'étroite parenté entre ces premières bandes dessinées et l'histoire illustrée de Busch datant de 1865, traduite en américain en 1870 .

7 Dans le même temps, Busch a, d'une certaine façon, fait obstacle à l'arrivée de la bande dessinée moderne en Europe : en effet, compte tenu de la popularité de ses histoires illustrées, la bulle ne pouvait s'imposer contre le principe de narration classique qui faisait figurer les textes (le plus souvent en rimes) sous les images, ce à quoi tout le monde s'était habitué; pour les premières traductions de BD américaines comme The Katzenjammer Kids, Bringing Up Father ou bien Felix the Cat, les bulles furent retouchées au cœur même des cases et remplacées par du texte d'accompagnement sous les vignettes. Ce n'est qu'à partir de 1925 qu'Alain Saint-Ogan fut le premier en France et en Europe à faire de la bulle un constituant de la grammaire narrative de la BD dans sa série Zig et Puce.

8 Ainsi, les dessinateurs allemands qui, au début du $x^{e}$ siècle, brillèrent par leur art de l'illustration - Lyonel Feininger fut par exemple sollicité en 1906 par la Chicago Tribune et apporta sa contribution aux deux séries The Kinder Kids et Wee Willie Winkie's World, parues brièvement dans la presse - étaient certes nombreux, mais ils eurent quelque peine à adapter le principe narratif qui caractérise la bande dessinée moderne. À l'inverse des USA où la bulle, en l'espace de quelques années seulement, remplaça complètement les textes sous les images, l'association en un seul média du texte et du dessin fut tout d'abord ignorée dans le Vieux Monde; la forme narrative des histoires illustrées classiques resta de mise dans les magazines satiriques et les revues pour enfants.

9 Cela ne changea guère jusqu'en 1945, car à partir de 1933, sous le national-socialisme, l'Allemagne ne fit que s'isoler davantage afin d'échapper à toutes les influences culturelles possibles. Pour les nazis, les BD étaient des «sous-produits de la presse, plutôt ignorés en Europe, vu que le niveau mental de leur contenu était tout juste bon pour des enfants de dix ans et que le caractère primitif de ce qui est montré et représenté ne saurait suffire même aux hommes les plus simples de notre continent » 
(comme on pouvait le lire en 1944 dans un article du Illustrierter Beobachter). Parmi les rares exceptions, Vater und Sohn de Plauen, une référence toujours présente quand on parle de la BD allemande. Plauen s'appelait en réalité Erich Ohser (né à Plauen en 1903), il devait travailler sous un pseudonyme, car il était tombé en disgrâce auprès des nazis à cause de ses dessins critiques pour le journal social-démocrateVorwärts et avait été exclu du Reichsverband der deutschen Presse, ce qui équivalait à une interdiction professionnelle. Grâce à l'intervention d'Erich Kästner, il put néanmoins à partir de 1934 réaliser la magistrale série Vater und Sohn pour la Berliner Illustrirte Zeitung,

Ses historiettes pleines de chaleur et de poésie relatant les événements qui marquent la vie d'un bon père de famille tout en rondeur et de son fils (à qui Christian, le fils de Ohser avait servi de modèle) sont conçus comme une pantomime sans texte; elles constituent, par leur légèreté graphique, la première bande dessinée allemande qui rompt avec l'esthétique lourde des histoires illustrées classiques, et marquent une modernisation du récit illustré en Allemagne. Les deux personnages furent vite si populaires que leur notoriété fut utilisée pour la publicité et finalement, ils furent même récupérés par les nazis ;

11 C'est pour cette raison que Ohser mit fin à sa série en 1937. Il fut dénoncé et arrêté début 1944 pour " propos hostiles envers l'état ». Comme il savait ce qui l'attendait à la «Cour de justice du peuple » de Freisler, il se suicida dans sa cellule le matin du 6 avril, juste avant l'audience.

12 Après la Libération, les enfants découvrirent la BD essentiellement par l'entremise des GI américains. Dans les villes en ruines, de petites imprimeries au fond des caves commencèrent à produire leurs propres magazines. Mais c'est Micky Maus, la première publication en Allemagne totalement en couleurs et éditée par la filiale du détenteur de la licence Disney tout juste fondée au Danemark, qui fut dès 1951 le premier magazine à succès. En 1953, la maison d'édition Lehning à Hanovre entreprit de traduire des séries italiennes interminables comme Akim dans le format de «comic albums». Ces albums étaient extrêmement convoités en raison leur prix modique de deux Groschen (deux sous), et comme cela revenait moins cher à Lehning de faire dessiner ses propres histoires que de payer des droits de licence aux détenteurs des droits italiens, il se lança dans la production de ses propres séries.

Le dessinateur le plus important de cette maison d'édition fut Hansrudi Wäscher. Il était né en 1928 à Lugano, avait grandi au milieu des BD italiennes et les techniques narratives de ce medium lui étaient familières. Très vite, la première création de Wäscher, Sigurd, une histoire de chevalerie, fut un succès durable et parut de 1953 à 1963 en 581 albums. Suivirent de nombreux autres albums paraissant parallèlement chaque semaine, comme Tibor, Falk, une imitation d'Akim, également une saga de chevalerie, ou bien après le lancement de Sputnik en 1957 la série de science-fiction Nick der Weltraumfahrer. La principale spécialité de Wäscher étaient des récits d'aventures bien ficelés selon les clichés du genre et des intrigues qui restent en suspens à la fin de chaque album. Des collectionneurs ont rapporté qu'il avait dessiné quelques 22.291 albums entre 1953 et 1966. Ainsi a-t-il marqué considérablement la jeunesse d'aprèsguerre ; aujourd'hui encore, à 82 ans, il continue de dessiner; d'autres dessinateurs ont même pris la relève pour continuer à faire vivre ses personnages pour ses fans qui l'ont connu dans les ruines de l'après-guerre et ces mêmes artistes ont édité quelques-unes de ses séries en tirage de collection limité. 

au début des années cinquante dans les illustrés et qui parurent pendant plusieurs années. En tête arrivait Nick Knatterton que Manfred Schmidt commença à publier dans Quick en 1950, avec l'intention manifeste de parodier les bandes dessinées américaines. Très vite son astucieux détective au menton anguleux devint si populaire que ses aventures sont aujourd'hui encore reproduites dans certains ouvrages et ont même servi de document de base pour des dessins animés ou des films avec des acteurs réels. L'année suivante vit la naissance, dans le magazine de télévision Hör zu, de la série Mecki, avec ses histoires merveilleuses autour d'un hérisson et toutes sortes d'autres animaux personnifiés, dans lesquelles les dessinateurs Reinhold Escher et le «professeur » Wilhelm Petersen (qui intervenaient en alternance) renonçaient une fois de plus aux bulles et plaçaient sous les images des textes à l'écriture très sérieuse. Mecki devint une figure culte et paraît aujourd'hui encore, dans Hör zu, entre-temps considérablement modernisée et redessinée par des artistes comme Volker Reiche ou Hansi Kiefensauer.Pour Stern en revanche, Roland Kohlsaat dessina de 1953 à 1977 l'odyssée fantastique de Jimmy, das Gummipferd qui rapporte les voyages et aventures d'un gaucho et de son cheval en caoutchouc gonflable

Mais la bourgeoisie cultivée traita bientôt les magazines de BD de «sous-littérature pour analphabètes » et de « poison anti-littéraire et anti-pédagogique ». L'été 1953, une «loi sur la diffusion d'écrits constituant une menace pour la jeunesse » entra en vigueur, qui, tout comme aux USA au même moment, provoqua une 'autocensure volontaire' chez les éditeurs. Dans des réimpressions de Sigurd et dans d'autres séries, les adversaires du héros mouraient dorénavant tout à fait incidemment, sans qu'on ne pût apercevoir ni épée ni arme d'aucune sorte. jeunesse était Fix \& Foxi de Rolf Kauka. Kauka avait rassemblé autour de lui quelques dessinateurs pour réaliser des dessins animés, mais faute de moyens techniques, il se résigna en 1953 à faire un magazine de BD qui devint Fix \& Foxi. Au début, ses animaux étaient conçus comme des personnages fabuleux, mais très vite, il prit le parti de les styliser en s'inspirant de Walt Disney. Les deux renards Fix et Foxi (de même que Lupo, le chien, Knox, l'inventeur au bec de corbeau, et bien d'autres personnages) firent de Kauka un millionnaire (il mourut en 2000 à 83 ans, propriétaire terrien aux USA); malgré son esprit petit-bourgeois parfois passablement gênant Fix \& Foxi survécut jusqu'en 1994, année où le magazine cessa de paraître. Depuis, plusieurs tentatives pour donner un nouveau départ à cette célèbre galerie de personnages se sont soldées par un échec.

Les enfants de la «zone d'occupation soviétique » réclamaient eux aussi les bandes dessinées d'Allemagne de l'Ouest. «Il est évident qu'avec ces torchons, on vise à agresser et à abrutir une jeunesse pour la rendre complètement sourde aux menaces d'une guerre programmée ", prétendait le quotidien Neues Deutschland s'élevant ainsi contre ces « déchets impérialistes ». En RDA comme en Allemagne de l'Ouest, on put assister à des autodafés de BD, essentiellement pour des raisons idéologiques et non en invoquant le caractère bourgeois de la pédagogie comme à l'Ouest. En 1955 furent créées sur décision du «Comité Central de la FDJ » les deux magazines illustrés Atze et Mosaik qui devaient offrir une alternative dans la perspective d'une éducation socialiste. 
les aventures épiques de trois Digedags comme des héros de séries. Suivant les instructions de la maison d'édition Neues Leben, le dessinateur Hannes Hegen était tenu de créer un "contre-modèle » à Micky Maus. Alors que dans Atze, un magazine au caractèreplutôt corseté, de jeunes pionniers sacrifiaient héroïquement leurs vacances pour fonder une "Allemagne nouvelle», Dig, Dag et Digedag voyageaient de par le monde et traversaient l'histoire; ainsi en 1958, l'année qui suivit le lancement du spoutnik, ils firent même une excursion dans l'espace, où l'Union Républicaine, un état qui vivait en paix sur la planète Néon, se faisait assiéger par un Empire impérialiste, Grand-Néon. Mais en 1975, Hegen ne supporta plus l'emprise de l'idéologie sur la culture et se retira; Mosaik remplaça, pour des raisons de droits d'auteurs, les Digedags par les Abrafaxes.

19 Alors que la bande dessinée en RDA ne connaissait pas vraiment d'évolution jusqu'à la chute du Mur (les trois Abrafaxes restent aujourd'hui encore parmi les personnages de BD les plus populaires dans l'Est de l'Allemagne, voire dans plusieurs pays d'Europe de l'Est et d'Asie orientale où ils ont aussi paru), on assista, dans les années 70, au début d'une ère moderne de la BD en Allemagne fédérale. En 1972, Zack fut le premier magazine hebdomadaire de BD inspiré de Tintin en Belgique et ce sont essentiellement des séries d'aventures franco-belges qui remplacèrent les séries habituelles dont les dessins étaient produits en série et non signés.

201973 marqua aussi le début de la série Turi und Tolk dans Zack, sous la plume du dessinateur allemand Dieter Kalenbach. Kalenbach inscrivit sa série dans une tradition essentiellement francophone en lui donnant un caractère éminemment naturaliste (le scénario tournait autour d'un jeune Lapon et de son aigle); il fut le premier artiste de $\mathrm{BD}$ à se détacher complètement de tout caractère national et à se présenter comme appartenant à une culture européenne de la bande dessinée. L'année suivante commença à paraître Comixene, une revue spécialisée qui existe encore de nos jours et qui avait pour ambition de se hisser au niveau international en collant au plus près des évolutions en cours.

21 Autre fait notable, la jeune génération développa, dans la foulée des mouvements étudiants de 1968, une conception nouvelle, anarchiste de la vie. Elle découvrit Barbarella, Asterix et Robert Crumb. Sous la plume de Gerhard Seyfried, les freaks et les «flics» aux nez bulbeux se retrouvaient bientôt sur quasiment tous les tracts de la scène alternative de gauche. Dès sa parution en 1981, Invasion aus dem Alltag (Invasion du quotidien), la première BDdeSeyfried, correspondait complètement à l'esprit révolutionnaire de l'époque, devint un best-seller et se vendit à plus de 100000 exemplaires. Encore plus populaire que les gros nez de Seyfried fut son contemporain Werner, du dessinateur Brösel (c'est-à-dire Rötger Feldmann), une série qui mettait en scène un prolo, toujours sur sa moto, en train de siroter une bière. Des revues de BD alternatives comme Zomix et Hinz \& Kunz apparurent et devinrent un champ d'expérimentation pour une nouvelle génération de dessinateurs comme par exemple Tomas M. Bunk, qui habite aujourd'hui à New York et qui travaille pour le magazine satirique américain Mad. Les premières bandes dessinées féministes de Marie Marcks et Franziska Becker furent publiées dans des magazines bien dans l'esprit du temps et en 1981 parut le premier volume des Schwulcomix oùRalf König croque ses homos à gros nez. 

influencés par la BD de l'underground américain que se développa la BD allemande des années 1980; elles furent d'ailleurs perçues comme telles par un large public. Et le cinéma a fait le reste. Der bewegte Mann (Les nouveaux mecs),inspiré de la BD éponyme de Ralf König parue en 1987, devint en 1994, avec six millions et demi de spectateurs, le deuxième grand succès allemand de l'histoire du cinéma; le film fut projeté dans 47 pays et récompensé en 1995 par le Prix du film allemand. (Entre-temps, Kondom des Grauens (La capote qui tue), Wie die Karnickels (Comme des lapins) et Lysistrata, de König, ont été également adaptés pour le cinéma. Trois autres adaptations sont actuellement en préparation). À leur suite sortirent quatre films inspirés du Werner de Brösel. Et puis il y eut aussi Das kleine Arschloch (Le p'tit con), de Walter Moers, une série de BD audacieuse qui vit le jour en 1990. Son héros : un nain malfaisant avec des yeux globuleux, affublé d'un nez monstrueux et d'une forte gueule déversant un flot d'obscénités. Cette BD ne passa que deux fois à l'écran et ne remporta qu'un succès relatif, l'humour anarchiste de Moers se révélant très peu compatible avec le cinéma.

En 1998, Moers réussissait en revanche un coup de génie avec sa bande dessinée Adolf, où il transposait à notre époque le personnage d'Hitler en être falot et ridicule. Malgré l'immense succès qu'il avait rencontré, Moers se retira de la BD et se consacra totalement à l'écriture de romans fantastiques et satiriques qui devinrent également des bestsellers. Entre-temps, les humoristes de BD qui avaient été populaires autrefois ont été un peu oubliés ; il en alla ainsi pour Gerhard Seyfried qui, avec la disparition de la contre-culture alternative, n'avait plus matière à dessiner ni, de ce fait, de lecteurs ; il préfère aujourd'hui écrire des romans. Au fil des années, le Werner de Brösel a aussi perdu de son mordant, et on ne le voit plus guère apparaitre que dans une publicité pour une compagnie pétrolière.

Ralf König est le seul à être resté fidèle à ce médium avec une impressionnante production régulière (jusqu'à quarante albums); il est aujourd'hui le plus célèbre des dessinateurs de BD allemands et celui qui connaît le plus de succès sur le plan international avec des traductions dans plus de quinze pays, y compris en Pologne, Lettonie et Croatie. Il faut dire qu'il a commencé à dessiner ses histoires de bonshommes gays à un moment où l'homosexualité était un sujet de société encore marginal et quasiment tabou. "Lorsqu'à la fin des années soixante dix on révélait à quelqu'un son homosexualité, dans le meilleur des cas, celui-ci vous regardait avec commisération, plissant le front d'un air profondément affecté »; c'est ainsi que König décrit l'atmosphère de l'époque où il dessina ses premières $\mathrm{BD}$, au moment de son propre coming-out. «Et puis soudain, on n'eut plus honte de son homosexualité, mais au contraire on en plaisantait. C'était nouveau, c'était le ton juste au bon moment». Aujourd'hui, les homosexuels en Allemagne peuvent se marier, les deux plus grandes villes d'Allemagne sont dirigées par des maires homosexuels, le pays a un ministre des Affaires Étrangères qui revendique son homosexualité; la libéralisation de la société qui s'exprime de cette façon est due notamment aux bandes dessinées de Ralf König. C'est surtout avec des albums comme Superparadise traitant du Sida (1999) ou Sie dürfen sich jetzt küssen sur les «mariages homos" (2003) qu'il se fit le chroniqueur de la vie quotidienne des homosexuels. À la question de savoir si ses bandes dessinées n'étaient pas parfois à la limite de la pornographie, Ralf König répond simplement : «Comment des personnages qui ont des nez plus gros qu'une bite peuvent-ils être pornographiques?» 

aussi ouvertement, avec la même franchise et le même courage que son homosexualité. Dschinn Dschinn (Djinn Djinn) fut la première bande dessinée, et avec plus de 300 pages la plus volumineuse à ce jour, à traiter l'hostilité au plaisir sexuel de l'islamisme radical. « La contradiction qui existe entre l'Orient sensuel des Contes des Mille et une Nuits et la condamnation du plaisir sexuel par les Talibans, avec le port de la barbe obligatoire, l'interdiction de la musique, la disparition des femmes sous la burqa etc., m'a toujours étonné ». Il était en train de travailler à son premier volume quand, le 2 novembre 2004, survint l'assassinat sauvage du metteur en scène et écrivain satirique Theo Van Gogh à Amsterdam. Peu avant qu'il ne termine son deuxième volume éclata, début 2006, la polémique autour des caricatures de Mahomet, où tout à coup des dessinateurs danois virent leurs têtes mises à prix, et où des ambassades, entre autres à Casablanca et Karachi, furent incendiées dans des émeutes entraînant la mort de deux cents personnes. liberté de pensée et donc de ce fait à sa liberté en tant qu'artiste. "Comment peut-on encore appréhender en tant qu'acteur culturel le sujet de l'islam si tout est susceptible d'être interprété autrement? Je me pose la question de savoir si je peux employer maintenant dans une bulle des termes aussi brûlants que "charia", "coran" ou "Allah" ? Est-ce que je peux me le permettre ou non, ici, au cœur de l'Europe?». Le malaise de König ne concerne toutefois pas que les fanatiques islamistes, mais aussi les milieux conservateurs de l'église de son propre pays qui, très vite, manifestèrent hypocritement une "certaine compréhension » et qui déclarèrent "qu'il fallait cesser de plaisanter avec la foi. Si on avait laissé, au cours des siècles, dit Knigge, les maîtres du Vatican agir comme ils le voulaient, l'Occident aurait lui aussi sombré dans l'obscurantisme. Le pire serait de laisser n'importe quel fanatique imposer son dieu par la force, en s'interdisant toute critique ou toute satire ". C'est dans ce contexte que parut entre 2008 et 2010 sa «Bibel-Trilogie », où König raconte sa propre version de la genèse dans les albums intitulés Prototyp (Prototype), Archetyp et Antityp. Il y dessine Noé en Taliban hostile au plaisir, totalement dépourvu d'humour, et qui finit par chapitrer sévèrement l'apôtre Paul.

auteurs s'illustrant dans la BD épique dont le renouveau est marqué par les premières publications de Matthias Schultheiss dans les années 1980 sont toujours un peu éclipsés par les dessinateurs de bandes dessinées humoristiques. Après le roadmovie Trucker qui se situait complètement dans l'esprit de Dieter Kalenbach et de son style inspiré des BD d'aventures franco-belges, on vit apparaître des histoires courtes inspirées de Charles Bukowski telles que les deux trilogies Die Wahrheit über Shelby et Die Haie von Lagos qui rencontrèrent un large écho sur le plan international. En 1992, Schultheiss eut l'occasion de participer au Salon international de la BD d'Angoulême, en France, où il exposa son travail et l'année suivante, il composa la série Propellerman pour la maison d'édition américaine Dark Horse. La carrière internationale de Schultheiss démontre toutefois qu'un BDiste allemand ne peut guère vivre de son art dans le contexte d'un marché encore aussi fluctuant. C'est d'ailleurs pour la maison d'édition française Glénat qu'il a réalisé sa dernière œuvre, Reise mit Bill (Le voyage avec Bill), un roman graphique qui compte pas moins que 300 pages.

Et pourtant, une nouvelle génération de dessinateurs peu effrayée par la médiocrité des perspectives économiques est entre-temps apparue en Allemagne. Il convient 
d'évoquer ici Isabel Kreitz qui peut se targuer d'une œuvre imposante, notamment avec son dernier album Die Sache mit Sorge, un récit de 250 pages sur Richard Sorge, espion de Staline, qui, en 1942, avait prédit au jour près mais en vain - puisque l'on n'en tint pas compte - l'attaque de l'Union Soviétique par la Wehrmacht allemande. Cet album rencontra un certain succès en France et en Espagne.

C'est surtout par sa polyvalence que Kreitz impressionne. Elle dessine aussi bien avec un humour persuasif que dans un style naturaliste méticuleux, elle a travaillé aussi bien pour des grandes que pour des petites maisons d'édition, pour des adultes aussi bien que pour des enfants, pour des quotidiens aussi bien que pour des magazines prestigieux, en noir et blanc comme en couleurs, elle dessine des comic-strips, des albums, des romans graphiques, elle travaille parfois avec des auteurs, adapte des romans (comme Uwe Timm ou Erich Kästner) ou encore elle écrit ses propres histoires. Cette année est prévue la publication de Haarmann, imposant roman graphique à l'atmosphère dense d'après un scénario de Peer Meter, consacré à l'indicateur de police et meurtrier en série du même nom qui sévit à Hanovre dans les années 1920.

Si l'Allemagne a été longtemps un pays où les éditeurs étrangers ne pensaient pas spécialement découvrir de nouveaux talents, la situation a fondamentalement changé ces dernières années. Et il n'est pas rare que les BDistes assistent à la première édition de leurs œuvres dans la France voisine. Ainsi Jens Harder avec Alpha. Directions, qui fut couronné du prix Max-und-Moritz comme la meilleure publication de BD de langue allemande au Salon international de la bande dessinée d'Erlangen: un album titanesque, publié dans sa version originale chez Actes Sud, en France, dans lequel Harder ne raconte rien de moins que l'histoire du cosmos, de la terre et de l'évolution de l'Homme et - comme souligné lors de la remise du prix - « retraitant graphiquement les modèles de la physique et de l'astrophysique, les prises de vue satellites, les découvertes préhistoriques des géologues et archéologues concernant les figures de sable des Navajos, les illustrations des Testaments bibliques, les représentations de Bouddha, Vishnu et de la Grande déesse, tout cela combiné avec quantité d'autres références culturelles et historiques ». À la fin, 350 pages plus loin, on assiste à l'éveil de l'humanité qui vient tout juste de naître - Harder prévoit encore deux, voire trois autres tomes qui devraient nous mener à l'époque contemporaine.

31 C'est avant tout son ouverture, son plaisir à expérimenter de nouveaux champs et sa diversité stylistique qui caractérise aujourd'hui la bande dessinée allemande - une manière d'appréhender leur pratique que de nombreux dessinateurs trouvent dans les modèles fournis par la maison d'édition française l'Association. Reinhard Kleist se fit connaître avec sa biographie de Johnny Cash, I see a darkness, il fit un voyage à Cuba, rédigea un journal de bord sous forme de carnet de croquis, Havanna, et travaille actuellement à une biographie de Fidel Castro. D'Arne Bellstorf, on attend pour cette année la parution de la biographie de Stuart Sutcliffe, ce membre fondateur des Beatles maintenant tombé dans l'oubli qui quitta le groupe en 1961, juste avant que les Beatles ne deviennent des stars à Hambourg. Le héros de Uli Oesterle, Hector Umbra, mène une existence ratée, cherchant à donner un sens à sa vie: son errance le conduit dans des bistrots déliquescents et enfumés, des catacombes obscures et des gouffres psychiques ; Oesterle, usant d'un art fracassant du dessin, dresse un portrait d'un Munich underground, où l'on se soûle de Jägermeister, de techno-beats et de drogues branchées au milieu de clochards prophétisant la fin du monde, comme on n'aurait jamais $\mathrm{pu}$ l'imaginer derrière les façades soignées de la Frauenkirche et de la Hofbräuhaus. Anke 
Feuchtenberger, née à Berlin-Est et aujourd'hui professeur à l'École supérieure de Sciences Appliquées de Hambourg au département « Illustration », se fit un nom avant tout par des albums aux dessins merveilleusement irréels et inquiétants comme Die Hure $H$ (La putain P), d'après des scénarios de Katrin de Vries.

Les dessinateurs trouvent aussi leur matière première dans leur propre biographie, à l'instar des auteurs de L'Association. Dans Wir können ja Freunde bleiben (On peut toujours rester amis) ou bien Die Band (The Band), Mawil (c'est-à-dire Markus Witzel) raconte d'un trait fluide et léger des histoires extrêmement personnelles du temps de sa jeunesse. Dans son roman graphique de 450 pages, Heute ist der letzte Tag vom Rest deines Lebens (Trop n'est pas assez), Ulli Lust raconte ses tribulations de punkette en Sicile, et Andi Lirium (évidemment un pseudonyme) raconte dans Punkrock Heartland les sentiments d'amour et la haine que partageaient les squatters de Hambourg. Vingt ans après la chute du Mur, Claire Lenkova (Grenzgebiete) et Simon Schwarz (Drüben) se souviennent de leur enfance dans une Allemagne divisée. Flix (c'est-à-dire Felix Görmann) a créé avec Heldentage un journal sous forme de BD dans lequel il a consigné au jour le jour, en un «strip» quotidien, tout ce qu'il a vécu et ressenti, autrement dit les "petites aventures du quotidien »; il est allé plus loin encore dans Held, où il a poursuivi sa biographie dessinée jusqu'en 2064. De son côté, Lile Hoven, fille d'une Américaine et d'un père allemand, réinvente ses propres racines dans Liebe schaut weg en remontant dans la mémoire des familles de ses deux parents, par-delà les continents; Cet album, artistiquement dessiné avec la technique de la carte à gratter, s'achève par la naissance de son auteur.

33 Ces albums de facture le plus souvent avant-gardiste et d'une grande originalité visuelle paraissent chez différents petits éditeurs très engagés et s'efforçant de promouvoir la bande dessinée comme forme d'expression artistique; on y trouve, en nombre croissant, des dessinateurs et dessinatrices allemands qui auparavant n'avaient aucune possibilité de se faire publier. Ce sont des éditeurs comme Reprodukt, Avant Verlag ou Édition Moderne qui, par le passé, tiraient rarement à plus de trois mille exemplaires. La production prolifique d'albums de BD ou de romans graphiques de ces derniers temps a cependant suscité l'intérêt croissant des chroniqueurs et aujourd'hui, les comptes rendus substantiels dans la presse quotidienne ou même des traductions à l'étranger ne sont pas rares.

Indéniablement, la presse joue actuellement un rôle important de focalisateur dans l'intérêt du public pour ce genre. De plus en plus de quotidiens publient leurs propres $\mathrm{BD}$ ou reproduisent des $\mathrm{BD}$ à titre expérimental. Laplutôt conservatrice Frankfurter Allgemeine Zeitung, qui n'avait encore jamais voulu publier de comptes rendus de BD fut le précurseur en 2002. On découvrit cette année-là avec Strizz, de Volker Reich, une sorte de soap opéra plein d'esprit, réunissant un employé de bureau du même nom et d'autres personnages commentant interminablement l'actualité brûlante de la vie quotidienne et politique; ces planches, une fois rassemblées - elles sont également disponibles en albums paraissant chaque année -, se lisent comme une chronique spirituelle et extrêmement distrayante du Zeitgeist de la République fédérale. Nous noterons également le Tagesspiegel berlinois qui a fait le choix éditorial d'accorder chaque semaine, dans ses éditions du dimanche, une page en alternance à des dessinateurs tels que Arne Bellstorf, Mawil, Flix et Tim Dinter. Ce projet contribuera, certes, à améliorer la situation financière de ces artistes, mais au-delà, il permettra 
d'assurer une présence nouvelle et générale à ce médium tout en répondant à l'intérêt croissant que porte le public aux romans graphiques d'artistes allemands.

Autre tendance actuelle: les écoles supérieures et les universités accordent depuis quelques années de plus en plus d'intérêt aux domaines de la bande dessinée, de l'animation et aux différentes formes de la communication par le design. Et déjà on voit apparaître dans le milieu estudiantin et universitaire des périodiques comme Strichnin ou Triebwerk ainsi que de minuscules maisons d'édition comme Mami qui publient des travaux de fin de semestre et mémoires de fin d'études. L'ensemble de ces publications est réuni au Jeune Forum du Salon International de la BD qui se tient tous les deux ans à Erlangen (depuis 2008 sont récompensées les premières publications d'étudiants) : c'est indéniablement le meilleur endroit pour se faire une idée de l'évolution de la BD made in Germany... Quel que soit son avenir, elle se trouve aujourd'hui à des années lumière de Wilhelm Busch, du hérisson Mecki et des renards Fix et Foxi.

Lors du Salon de Erlangen, le meilleur artiste de BD germanophone reçoit le prix Maxund-Moritz. Voici les primés :

\section{BIBLIOGRAPHIE}

1984 : Chris Scheuer

1986 : Matthias Schultheiss

1988 : Franziska Becker

1990 : Gerhard Seyfried

1992 : Ralf König

1994 : Hendrik Dorgathen

1996 : Thomas Ott

1998 : Bernd Pfarr

2000 : Martin tom Dieck

2004 : Ulf K.

2006 : Volker Reiche

2008 : Anke Feuchtenberger

2010 : Nicolas Mahler.

\section{RÉSUMÉS}

L'aperçu de la bande dessinée allemande que propose Andreas C. Knigge nous mène de «l'ancêtre » Wilhelm Busch - inventeur au XIX ${ }^{\mathrm{e}}$ siècle d'un concept graphique qui devait ouvrir mais aussi entraver durablement l'évolution du « comic » en Allemagne - à Anke Feuchtenberger, 
Isabel Kreitz ou Ralf König. La découverte des bandes dessinées américaines, aujourd'hui des classiques du genre, après 1945 éveille un intérêt qui culmine une première fois dans une modernisation du genre au cours des années 1970. Mais c'est la réunification des deux Allemagnes en 1989 qui donnera la formidable impulsion qui fera émerger cette nouvelle génération de dessinateurs allemands dont les œuvres sont aujourd'hui reconnues par-delà les frontières des pays germanophones. De l'ancienne « sous-littérature pour illettrés " à la matière enseignée dans les universités : la bande dessinée allemande se caractérise aujourd'hui par un sens de l'ouverture, une disponibilité à l'expérimentation graphique et textuelle et une variété stylistique qui font d'elle une forme d'expression artistique à part entière.

Vom «Ahnvater» Wilhelm Busch, der im 19. Jhdt ein graphisches Konzept vorweggenommen, jedoch auch der Entwicklung des Comics in Deutschland in mancher Weise den Weg verstellt hatte, bis zu Anke Feuchtenberger, Isabel Kreitz oder Ralf König spannt Andreas C. Knigge den Bogen zum Thema «Comic in Deutschland». Erst nach der Entdeckung der inzwischen klassischen amerikanischen Comics nach 1945 wächst das Interesse an der Gattung, und lässt in den 70er Jahren eine Art «Comic-Moderne» entstehen. Nach der Wiedervereinigung im Jahre 1989 wuchs dann jene neue Generation deutscher Zeichner heran, deren Werke inzwischen zunehmend auch Aufmerksamkeit jenseits der Landesgrenzen finden und in Übersetzungen im Ausland erscheinen. Vom «Lesefutter für Analphabeten» zum an Hochschulen und Universitäten gelehrten Fach: die Offenheit, Experimentierfreude und stilistische Vielfalt des aktuellen deutschen Comics erhebt ihn zu einer anerkannten künstlerischen Ausdrucksform.

In his general survey of German comic books that goes all the way to Anke Feuchtenberger, Isabel Kreitz and Ralf König, Andreas C. Knigge starts with the «ancestor», Wilhelm Busch, who invented the $19^{\text {th }}$ century graphic concept that was to lastingly open up - as well as hamper - the evolution of comics in Germany. When American comic books, which have since become classics of the genre, were discovered after 1945, the interest they aroused helped modernise the genre for the first time during the 1970's. But the reunification of the two Germany's in 1989 gave a tremendous impetus to the new generation of German cartoonists whose works are acknowledged beyond the borders of German-speaking countries. From the old «sub-literature for illiterates» to material taught at universities: the German comic book has an openness, an availability to graphic and textual experimentation and a variety of styles that make it a fullyfledged artistic form of expression.

\section{AUTEURS}

\section{ANDREAS C. KNIGGE}

Spécialiste de la BD allemande co-fondateur de la revue Comixene 